\title{
Calculations of the effects of point defects on Hubbard models of high temperature superconductivity
}

\author{
J W HALLEY*, S DAVIS, P SAMSEL and ROBERT JOYNT ${ }^{\dagger}$ \\ School of Physics and Astronomy. University of Minnesota, Minneapolis, Minnesota 55455, \\ USA \\ ${ }^{\dagger}$ Department of Physics, University of Wisconsin, Madison, WI 53706, USA
}

\begin{abstract}
We describe studies of the effects of defects on Hubbard models of high $T_{\mathrm{c}}$ superconductors. We first present numerical mean field calculations on the $t-J$ model in which disorder due to point defects in treated explicitly. Secondly, we describe variational Monte Carlo calculations at zero temperature in which the full effects of the Gutzwiller projection are included in a calculation of the variational BCS ground state including randomly distributed point defects. Finally we discuss possible microscopic mechanisms by which point defects could enhance pairing in the BCS state.
\end{abstract}

Keywords. Point defects; Hubbard models; oxides.

\section{Introduction}

A conspicuous feature of the new superconductors (Bednorz and Muller 1986; Wu et al 1987) which is usually assumed to be an inessential complication by theorists is that virtually all of the high temperature materials have a very high degree of spatial disorder leading to mean free paths which are at best a few times the superconducting coherence length. This means at least that one should be developing theoretical methods and models for taking theoretical account of the microscopic disorder. There is also a possibility (Halley and Shore 1988a; Phillips 1987) that some kinds of point defects or twin boundaries might act like "pairing centres" and enhance the pairing interactions leading to the superconductivity itself. For both these reasons we have been developing both mean field and correlated calculational methods and models for taking account of the effects of point defects in the $t-J$ version of the Hubbard model.

\section{Mean field model}

We have focussed attention on model in which the disorder arises from a random distribution of $J$ values on the bonds of the $t-J$ model on a square lattice. Because we had in mind a model (Halley and Shore 1988a) in which the different values of $J$ arose from the presence of oxygen vacancies in the plane, we chose a binary distribution of $J$ values, with a larger value of $J$ on randomly selected bonds than on the rest of the lattice bonds. In our numerical work, the fraction of these bonds was small in keeping with reports of small (but finite) concentrations of oxygen vacancies in the planes of many materials. In earlier work we reported approximate

*For correspondence 


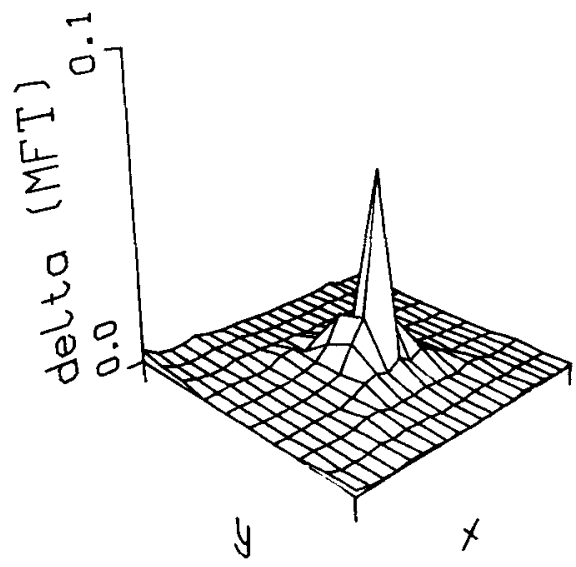

Figure 1. Magnitude of the gap function in mean field theory. $t=1$ and $J=1.75$ on the central $\mathbf{x}$-bond and zero elsewhere.

solutions of the mean field equations for such a system, in which the gap function as a function of "vacancy" concentration was calculated self-consistently in a method which took the disorder explicitly into account. The result, for a one-band model with one-electron orbital per site, was that a BCS-like state was stable in such a system, with a $T_{c}$ which varied with vacancy concentration $p$ and $J$ approximately as $\exp (-$ constant $/ J p)$. A physical picture of the nature of such a state is given in Halley and Shore (1988b). We have shown more recently (Halley et al 1990) that these qualitative results also occur in a two-band model in which the carriers are holes on "oxygen" orbitals, centred on the bonds of the square lattice. In figure 1, we show the local "susceptibility" calculated in the mean field theory of the one-band model. The pair function $\Delta_{i j}$ was fixed at a central bond, and the value of $\Delta_{i j}$ arising at neighbouring bonds was calculated using our equation-of-motion methods at neighbouring bonds. This local response function proves useful in the variational calculations on the full $t J$ model described in the next section.

\section{Variational calculation including Hubbard correlations}

To check whether a pairing state of the sort described in the preceding section survives the correlations induced by the large $U$ of the Hubbard model, we have made variational calculations on similarly disordered models by extending the work of Gros (1988) to disordered systems. We briefly review the method of Gros (1988). The variational wavefunction used in that work was of the form

$$
|\mathrm{RVB}\rangle \propto P|\mathrm{BCS}\rangle
$$

projected onto $N$ particle states

$$
|N\rangle \propto P\left(\sum_{\mathbf{k}} a(\mathbf{k}) c_{\mathbf{k}, \uparrow}^{\dagger} c_{-\mathbf{k}, 1}^{\dagger}\right)^{N / 2}|0\rangle
$$

Here $P$ is the Gutzwiller projection operator which forbids double occupancy at sites, 
$c_{\mathbf{k}, \uparrow}^{\dagger}$ is a creation operator for an electron in a plane wave state of wavevector $\mathbf{k}$ and

$$
a(\mathbf{k})=v_{\mathbf{k}} / u_{\mathbf{k}}=\Delta_{\mathbf{k}} /\left[\xi_{\mathbf{k}}+\left(\xi_{k}^{2}+\Delta_{\mathbf{k}}^{2}\right)^{1 / 2}\right]
$$

in which $\Delta_{k}$ is a variational function associated with the BCS gap and $\xi_{\mathbf{k}}$ is the one particle energy of an electron with wavevector $\mathbf{k}$ relative to the Fermi energy. The variational wavefunction $|N\rangle$ can be rewritten as

$$
|N\rangle \propto P\left(\sum_{j, j^{\prime}} a\left(\mathbf{R}_{j^{\prime}}, \mathbf{R}_{j^{\prime}}\right) c_{\mathbf{R} j, \uparrow}^{\dagger} c_{\mathbf{R} j^{\prime} \downarrow \downarrow}^{\dagger}\right)^{N / 2}|0\rangle,
$$

where $a\left(\mathbf{R}, \mathbf{R}^{\prime}\right)$ is the discrete fourier transform of $a(\mathbf{k})$. Gros developed a Monte Carlo technique for calculating the expectation value of the energy of the $t-J$ model with respect to this variational wavefunction. He found on an 82 site square lattice that a state with a $\Delta_{k}$ with $s$-wave symmetry was not stable with respect to the state with $\Delta_{\mathbf{k}}=0$ but that a $\Delta_{\mathbf{k}}$ with $d$-wave symmetry did have a lower energy.

To extend the variational method to a disordered system we write the one-electron eigenfunctions $\phi_{n}(\mathbf{r})$ of the disordered system in terms of tight binding functions $\phi_{n}(\mathbf{r})=\sum_{j} A_{n j} \phi\left(\mathbf{r}-\mathbf{R}_{j}\right)$. Then the appropriate generalization of $|N\rangle$ is obtained by using

$$
\begin{aligned}
& a\left(\mathbf{R}_{j}, \mathbf{R}_{j^{\prime}}\right)=\sum_{n} A_{n, j 1}^{*} A_{n, j 2} v_{n} / u_{n}, \\
& v_{n} / u_{n}=\Delta_{n} /\left[\xi_{n}+\left(\xi_{n}^{2}+\Delta_{n}^{2}\right)^{1 / 2}\right] .
\end{aligned}
$$

We have adopted a code used for the homogeneous case to this problem, calculating the eigenstates of the disordered one electron 82 site system by direct diagonalization. We studied three different variational forms for $\Delta_{n}$ :

$$
\begin{aligned}
& \Delta_{n}=\text { constant, } \\
& \Delta_{n}=\Delta_{0}, \xi_{n} \text { above the bottom of the band } \Delta_{1}, \text { otherwise, } \\
& \Delta_{n}=\sum_{i, j} A_{n, i}^{*} \Delta_{i, j}^{\mathrm{MFT}} A_{n, j} .
\end{aligned}
$$

In the first case we are neglecting any inhomogeneity induced in the gap function by the disorder. In the second case we try to account for such disorder by assuming that states lying below the lowest energy states of the ordered system have a different gap parameter. In the third case we choose the same functional form for the gap function which we found in the mean field calculation described in the previous section but let the amplitude of the function be a variational parameter. Specifically, we used the functional form arising from the calculational results shown in figure 1 , multiplied by a gap parameter $\Delta$. In the third case, we also took account of the fact (Halley and Shore 1988a) that the mean field calculation gave stable solutions only if the quantities $\Delta_{i j}$ had values of opposite sign on the $x$ and $y$ bonds of large $J$ respectively. If, in case 3 , the mean field solution had finite amplitude at a given bond arising from more than one defect site, then the gap functions from the mean field solutions were added to give the gap function for that bond. Results for an 82 site lattice in the three cases are shown in figure 2 . In the case of the first two variational forms, there were 8 holes ( 74 electrons) on the lattice and just one set of defects was used. In those two cases, there were 10 randomly distributed defect bonds on the 82 


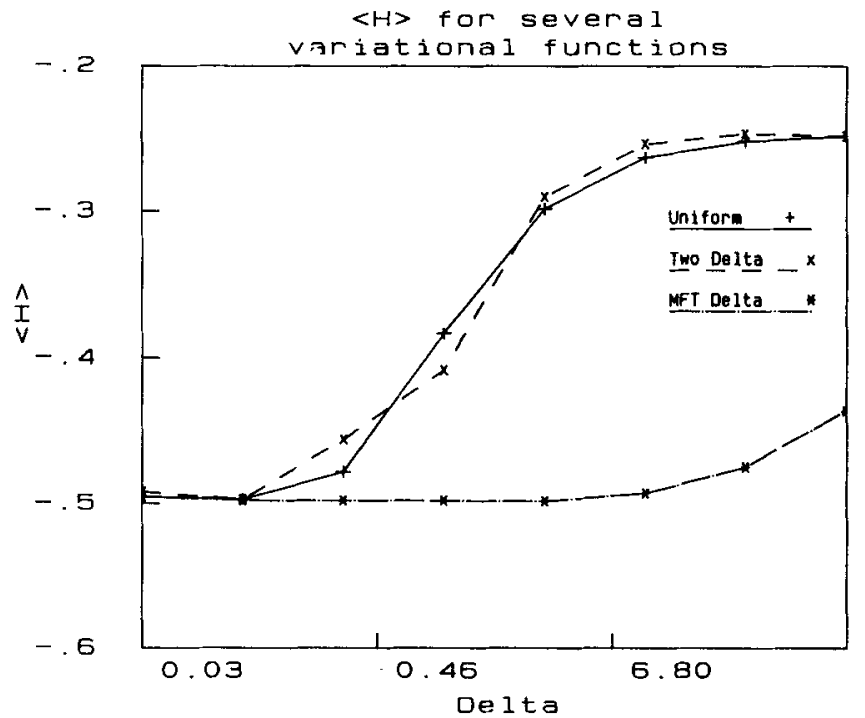

Figure 2. Variational results for the energy using the three types of variational wavefunction described in the text.

site lattice, corresponding to about $6 \%$ of the 164 bonds on the lattice. In the case of the third variational wavefunction, the number of holes was the same and we have averaged the results over 10 realizations of the defect structure model in which the defects are distributed randomly on bonds with a concentration of $5 \%$ of the total number of bonds (as in the percolation model). In the case of this third variational wavefunction, our estimate of the variance of the result shows that the error is less than the size of the points in the graph. We have made 3000 Monte Carlo moves per site (as in Gros 1988) for each realization with each variational wavefunction. The calculations for the third variational wavefunction required 40 hours with a fully vectorized code on a Cray XMP computer.

The results show clearly that the first two variational wavefunctions do not give a stable state with non-zero gap, but the third wavefunction does appear to do so. The value of $\Delta$ needed is quite large. One should bear in mind that this $\Delta$ is the value of the gap function at the defect bond. The actual superconducting gap would be much smaller. In these calculations we took $J=1$ at defect bonds and $J=0.25$ at other bonds. Energies are measured with respect to $t=1$. The diagonal energy at sites next to defect bonds was given the negative value of -0.5 and was zero elsewhere. The values of the energy shown do not include the expectation value of the term called $H_{\text {eff }}^{(3)}$ in Gros (1988). In the homogeneous case, this term had a much smaller expectation value than the other terms.

\section{Speculative discussion of possible pairing enhancement mechanisms by point defects}

These results suggest that the Hubbard correlations may not destroy the kind of defect induced pairing discussed in mean field theory (Halley and Shore 1988b). Here 
we briefly discuss some possible microscopic origins of such pairing enhancement by point defects.

One possibility, as discussed in Halley and Shore (1988b), is that oxygen vacancy defects could result in an effectively larger value of $J$ as in our numerical models. Here we describe a simple argument for this in the case of oxygen hole superconductors which is different from our earlier discussion: (We note that we make a sharp distinction between holes and vacancies here as a few theoretical authors do not. A vacancy is a missing oxygen atom. A hole is an oxygen atom with charge -1 instead of -2 .) As discussed by many authors, an oxygen vacancy is expected to bind two screening electrons, residing mainly at the copper ions on each side of the vacancy, thus rendering the valency of those copper ions $1+$. A free hole propagating through the lattice on the oxygen ions can approach the charge neutral vacancy without coulomb penalty and mix with a hole on one of the two $\mathrm{Cu}^{1+}$ ions next to it without expending energy $U$ (because the copper is cuprous). Thus holes will attain a larger $d$ orbital character as a consequence of the presence of oxygen vacancies. If one imagines that a second hole migrates to the second copper adjoining the vacancy (overcoming the coulomb barrier due to the first hole) then the two copper holes will interact with an exchange interaction across the space between them (which is empty of atoms because the oxygen is missing). The specific models described above were motivated by the idea that this exchange interaction might be large, because the copper ions relax toward each other and because the exchange is direct. Actual calculation of the exchange is a subtle quantum-chemical problem and we do not know if the exchange is large or not.

There are other possible candidates for pairing enhancement due to point defects. For example, consider an acceptor such as a $\mathrm{Sr}$ ion in $\mathrm{LaCu}_{2} \mathrm{O}_{4}$. The strontium is an acceptor. When ionized, it has a net negative charge relative to the undoped lattice. If the acceptor is screened by a hole, then it can be polarized by a passing free hole and the resulting polarization could attract another free hole moving with the appropriate phase, just as in the classical excitonic mechanisms. This is effective attraction through a particle-hole channel associated with the bound states of the acceptor. If the acceptor is ionized, then there is a corresponding hole-hole channel which could possibly result in attraction: Two holes might reside briefly on the acceptor centre, staying out of each others' way by moving so that the acceptor stays between them, somewhat as an $\mathrm{H}^{-}$ion is stabilized. Similar scenarios could clearly be constructed for donors.

These remarks obviously do not prove that point defects enhance pairing interactions but provide a possible starting point for detailed microscopic calculations to explore whether they could.

\section{Conclusions}

Our variational calculations indicate that the point defect enhanced pairing state discussed earlier in mean field theory can probably exist in the presence of the correlations which suppress charge fluctuations in the $t J$ model. Our methods also provide a means to study the effects of disorder on the $t J$ model in other models in which there is no pairing enhancement. We qualitatively discussed a few microscopic scenarios by which point defects might induce pairing. 


\section{Acknowledgements}

This work was supported in part by the Electric Power Research Institute and by the Minnesota Supercomputer Institute. Herb Shore is thanked for many discussions.

\section{References}

Bednorz J G and Müller K A 1986 Z. Phys. B64 189

Gros C 1988 Phys. Rev. B38 931

Halley J W and Shore H 1988a Phys. Rev. B37 525

Halley J W and Shore H 1988b in Theories of high temperature superconductivity, (ed.) J W Halley (Redwood City: Addison-Wesley) p. 115

Halley J W, Sen S and Davis S 1990 (unpublished)

Phillips J C 1987 Phys. Rev. Lett. 581028

Wu M K et al 1987 Phys. Rev. Lett. 58908 